\title{
An Efficient DTW Matching for On-line Signature Verification
}

\author{
Xinghua $\mathrm{Xia}^{1, \mathrm{a}^{*}}$, Dafang Yang ${ }^{1, \mathrm{~b}}$ and Fangjun Luan ${ }^{1, \mathrm{c}}$ \\ ${ }^{1}$ School of Information and Control Engineering, Shenyang JianZhu University, Shenyang, China \\ axxh8787@sjzu.edu.cn, ${ }^{\mathrm{b}}$ Yangdafang@sjzu.edu.cn, ${ }^{\mathrm{a}}$ luanfangjun@sjzu.edu.cn
}

Keywords: On-line signature verification; DTW; Signature curve constraint; Signature matching.

\begin{abstract}
On-line signature verification is one of widely acceptable biometrics. Dynamic time warping (DTW) is a nonlinear optimization method, which is widely used in on-line signature verification. DTW provides a computational technique of normalization and alignment to make best matching between two signatures, which might have different number of sample points. However, heavy computation is one of defects of DTW when sampled points included in signatures are increased. A simple and efficient method of DTW with signature curves location constraint (DTW with SCC) is proposed to improved efficiency of DTW matching. Several experiments are carried out on standard on-line signature dataset MCYT_Subcorpus_100 (DB1), which consists of 5000 signatures from 100 individuals in total. Experiment results demonstrate that the efficiency of on-line signature verification is improved greatly by our proposed method. The best result is given by $\mathrm{EER}=2.33 \%$, which also indicates the effectiveness of our proposed methods.
\end{abstract}

\section{Introduction}

Biometrics is an important field that allows personal identity verification through the analysis of personal characteristics. It is gaining popularity as a more trustable alternative to password based security systems. On-line signature verification is one of widely acceptable biometrics due to the fact that handwritten signatures have long been established during human daily life. It is difficult to be imitated and forged because signature will be unique and consistency for a given period. Moreover, technologies of signature verification require no invasive equipments and people are familiar with the use of signatures in daily life.

On-line signature verification can generally be divided into two groups, i.e. parametric and functional, according to features used in verification [1,2]. In parametric approach, a set of parameters or vectors are abstracted from sequence of signature, and the signature is represented by series of parameters. When functional approach is concerned, signature is usually characterized in terms of a time function, whose values constitute the feature set. Generally, it is less computation and more efficiency of parametric approach. When functional approach is concerned, it is heavy computation but higher accuracy of on-line signature verification. In verification process, the authenticity of test signature is evaluated by matching its features against those stored in knowledge base for given individual. There are some commonly used verification methods, such as template matching methods (e.g. Euclidean distance, dynamic time warping (DTW), etc.) $[3,4,5]$, statistical based methods (e.g. Mahalonobis distance, distances statistics, membership function, neural networks (NN), hidden markov model (HMM), support vector machine (SVM), etc.) [6,7,8], and structural based methods (e.g. string matching, tree matching, structural description graph analysis (SDG), etc.) $[9,10]$.

\section{Preprocessing}

Prior to on-line signature verification, the user should be familiar with the signature acquisition device and be required to input signature skillfully. On-line signature is captured in real-time through acquisition devices with a fixed sampled interval, thus on-line signatures are represented as time series.

There might be noises, distortion and variation during signature acquisition, signatures should be 
preprocessed before verification. At first, signatures should be smoothed to reduce the interrupted noises and distortion. Set signatures as $S(n)=\{(x(n), y(n)), n=1,2, \cdots, N\}$. Cubic polynomial is used to smooth and approximate signatures in our works. Afterwards, signatures are normalized to reduce fluctuations which are caused by signature position and size deviations. In our works, signatures are normalized,

$$
\hat{S}(n)=\frac{S(n)-S_{\min }}{S_{\max }-S_{\min }}
$$

where, $\hat{S}(n)=\{\hat{x}(n), \hat{y}(n)\}$ is normalized signature after normalized, $S_{\min }=\min S(n)$ and $S_{\max }=\max S(n)$. $\mathrm{N}$ is total number of sampled points of signature.

\section{DTW Matching with SCC}

When template matching approaches are considered, DTW is commonly used for signature matching. In traditional DTW algorithm, a full distance matrix, which includes all distances between sampled points contained in two signatures, should be calculated at first. Then the optimal path is planned by dynamic programming to obtain the minimum distance. It is heavy computation and inefficiency if sampled points included in signatures increased. We propose a modified method based on DTW with Signature Curves Constraint (DTW with SCC) for signatures matching. In DTW with SCC, features are not matched by DTW directly. Instead, features are matched with the location constraints, which are inherent in two matching signature curves. The location constraint can be obtained by optimal path, which is dynamic programming planned during the DTW matching of the two signature curves.

For a given individual, let $s(n)=\{(x(n), y(n)), n=1,2, \cdots, N\}$ and $t(m)=\{(x(m), y(m)), m=1,2, \cdots, M\}$ be reference and test signature curves respectively. $s(n)$ and $t(m)$ are matched by DTW at first, and optimal matching path is obtained by dynamic programming, i.e. $W_{(s, t),(x, y)}=\left\{w_{1}, \cdots, w_{r}, \cdots, w_{R}\right\}$, where, $r=1,2, \cdots, R, w_{r}=\left(w_{r_{s}}, w_{r_{t}}\right)$ denotes the matching pairs on the DTW path, which is called the SCC. $w_{r_{s}}$ and $w_{r_{t}}$ are matching points on the path included in reference and test signature curves respectively. $\mathrm{R}$ is the length of optimal path of DTW, and $\max (N, M) \leq R \leq N+M-1$. An example of SCC is also given in Fig. 1. With the optimal path of DTW having been planned, matching pairs on DTW path are obtained as $w_{r}$, the length of optimal path $\mathrm{R}=13$. Matching points on the path included in reference and test signature curves can be given as $w_{r_{s}}=\{1,2,3,3,3,4,5,6,7,8,9,10,11\}$ and $w_{r_{t}}=\{1,2,3,4,5,6,7,8,9,9,9,10,11\}$.

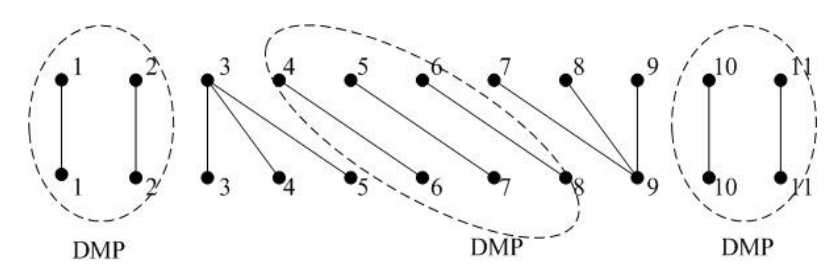

Figure1. DTW matching path of two sequences

With SCC, the similarity of feature $f_{k}$ between reference and test signature can be calculated,

$$
\text { Dist }=\sum_{r=1}^{R} \sqrt{\left(f_{k_{s}}\left(w_{r_{s}}\right)-f_{k_{t}}\left(w_{r_{t}}\right)\right)^{2}}
$$

According to the discussions above, not only the complete information of signature but also the DTW dynamic programming is included in the proposed method of signatures matching.

The efficiency of on-line signature verification can be improved by proposed method. The computational complexity of proposed method is given by $\mathrm{O}(N \cdot M+K \cdot(N+M-1)) \cong \mathrm{O}(N \cdot M)$ as opposed to $\mathrm{O}(K \cdot N \cdot M)$ of traditional DTW. Where, $K$ denotes feature numbers which are used in on-line signature verification, $\mathrm{N}$ and $\mathrm{M}$ denote the length of two signatures respectively. 


\section{Experiments}

Several experiments based on large scale dataset of MCYT_Subcorpus_100 (DB1) [11] are carried out. DB1 consists of 5000 on-line signatures from 100 individuals. For each individual, there are 50 signatures in all, out of these, 25 signatures are genuine and 25 signatures are skilled forgeries. 5 genuine signatures are selected randomly from genuine signatures to be used as references.

As discussed above, features do not matched by DTW directly for improving the efficiency. Alternatively, feature could be matched by SCC which is inherent in signature curves in our works. The optimal matching path of signature curves can be obtained by two signature curves matching with DTW, as shown in Fig. 2. The features matching with SCC are shown in Fig. 3. With the comparison, features matching with DTW are shown in Fig. 4. From the results, features can be matched well by our proposed method.

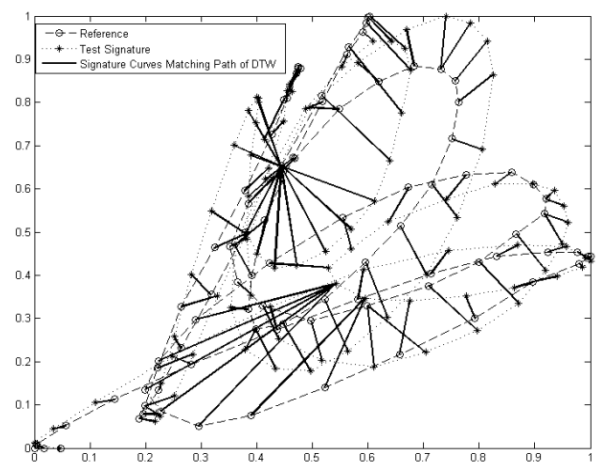

Figure 2. Signature curves matching path of DTW

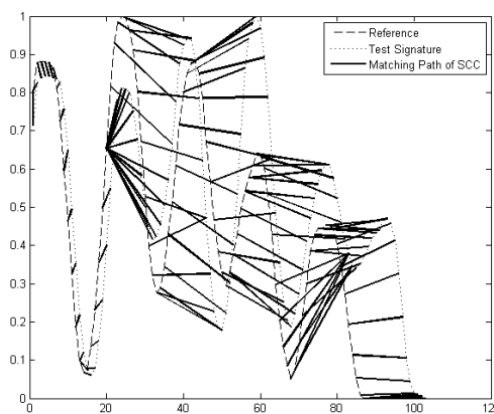

(a)

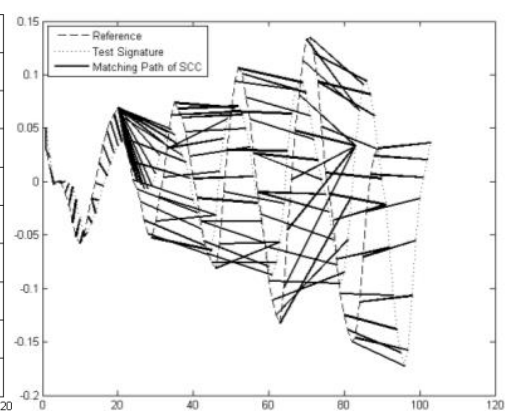

(b)

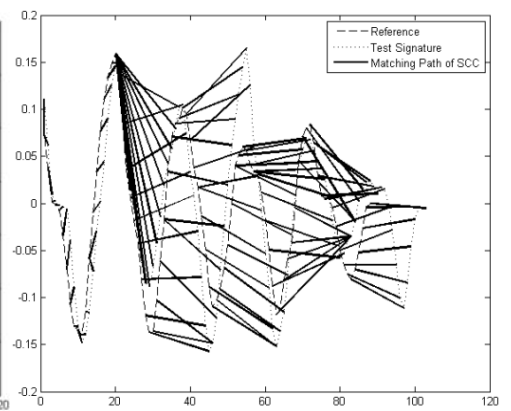

(c)

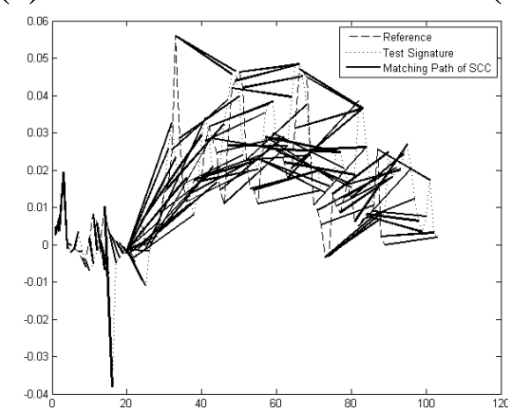

(d)

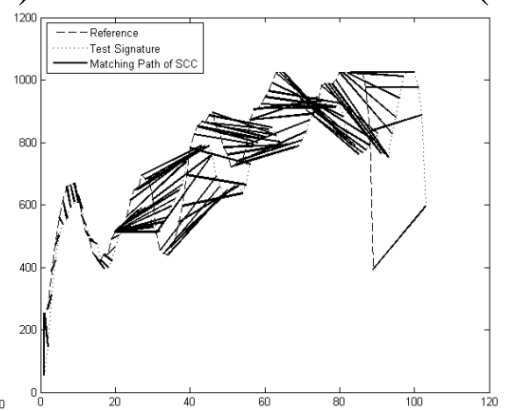

(e)

Figure 3. Signature features matching with SCC. (a) feature $Y$; (b) feature $V x$; (c) feature $V y$; (d) feature $a c ;(\mathrm{e})$ feature $P$ 


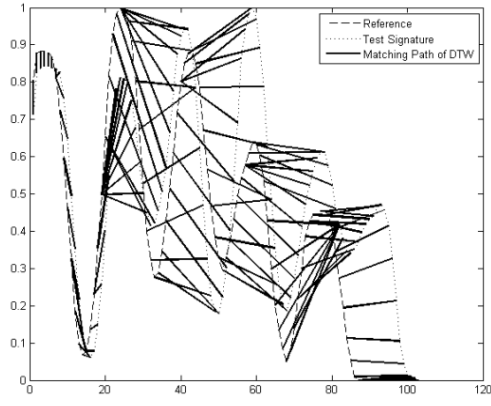

(a)

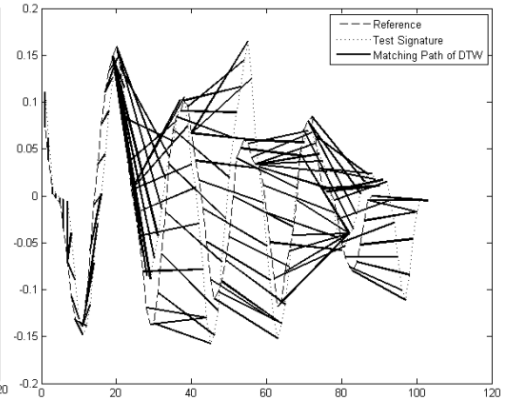

(b)

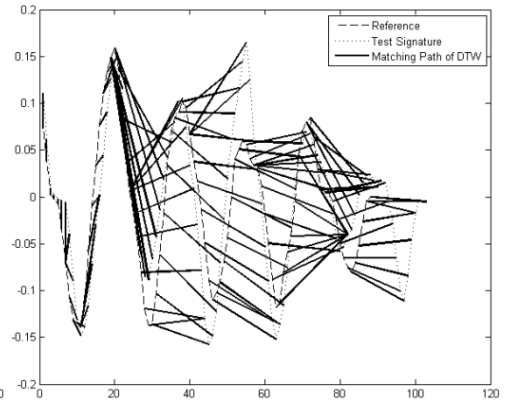

(c)

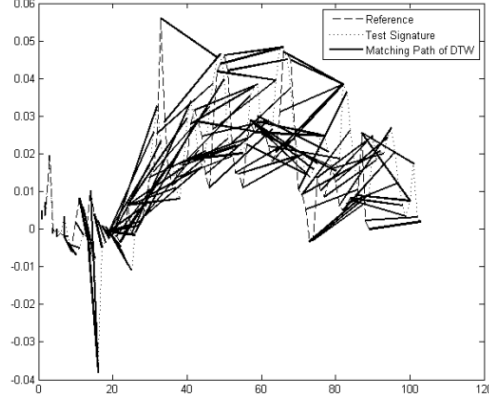

(d)

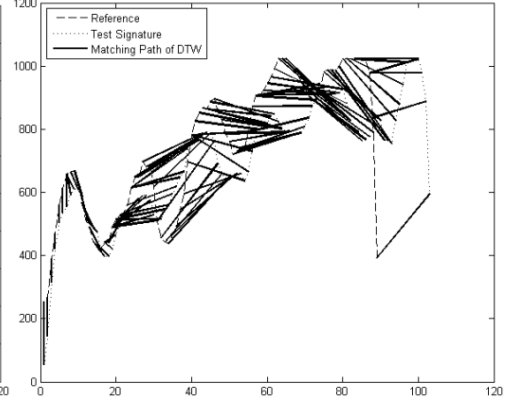

(e)

Figure 4. Signature features matching with DTW. (a) feature $Y$; (b) feature $V x$; (c) feature $V y$; (d) feature $a c ;(\mathrm{e})$ feature $P$

Time consumption of DTW and our proposed modified DTW with SCC are shown in Fig. 5. When one feature is used in verification, time consumption of DTW is $0.021 \mathrm{~s}$ which is lower than $0.025 \mathrm{~s}$ of DTW with SCC for the reason that the feature used in DTW is 1-dimension matching, while SCC used in DTW with SCC is 2-dimension matching. When feature number being increased, time consumption of DTW is increased dramatically, but time consumption of modified DTW with SCC is increased slowly. As discussed above, five features are used in our on-line signature verification, time consumption of DTW is $0.088 \mathrm{~s}$ as opposed to $0.026 \mathrm{~s}$ of modified DTW with SCC. ROC curves are given in Fig.6, EER of our proposed method of DTW with SCC are 2.33\% as opposed to 2.84\% given by DTW. The results illustrate that our proposed modified DTW with SCC can provide higher accuracy and more efficient of on-line signature verification.

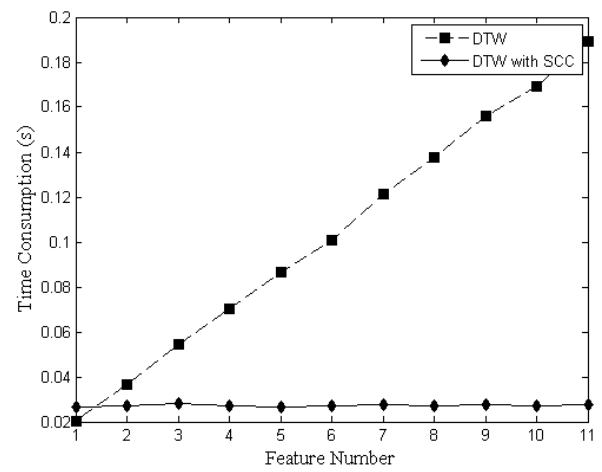

Figure 5. Time consumption 


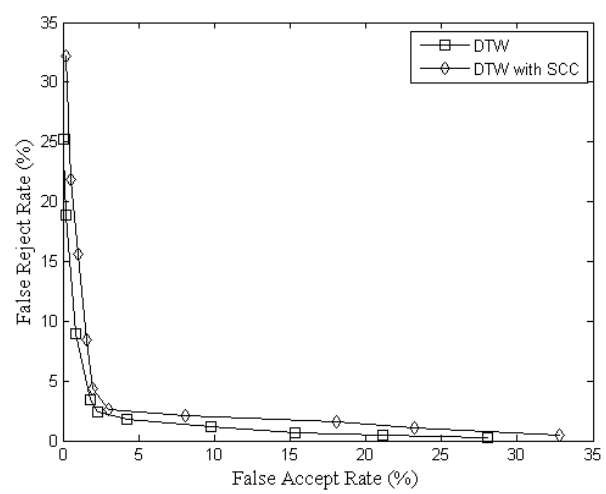

Figure 6. ROC curves

\section{Summary}

A simple method of efficient DTW matching for on-line signature verification was proposed in our paper. Contrast to conditional DTW used in signature feature's matching, the optimal planning path inherent in signature curves matching was employed to feature's matching. The experiment results demonstrated that both efficiency and accuracy of on-line signature verification were improved by our proposed method.

\section{References}

[1] Plamondon R, Srihari S, On-Line and Off-Line Handwriting Recognition: A Comprehensive Survey[J]. IEEE Transactions on Pattern Analysis and Machine Intelligence, 2000, 22(1): 63-84.

[2] Impedovo D, Pirlo G, Automatic Signature Verification: The State of The Art[J], IEEE Transactions on Systems, Man, and Cybernetics, Part C: Applications and Reviews, 2008, 38(5): 609-635.

[3] Reza A G, Lim H, Alam M J. An efficient online signature verification scheme using dynamic programming of string matching[M]. Convergence and Hybrid Information Technology. Springer Berlin Heidelberg, 2011: 590-597.

[4] Vivaracho-Pascual C, Faundez-Zanuy M, Pascual J M. An Efficient Low Cost Approach for On-Line Signature Recognition Based on Length Normalization and Fractional Distances[J], Pattern Recognition, 2009, 42(1): 183-193.

[5] Faundez-Zanuy M. On-Line Signature Recognition Based on VQ-DTW[J], Pattern Recognition, 2007, 40(3): 981-992.

[6] Thumwarin, Pitak, Jitawat Pernwong, and Takenobu Matsuura. FIR signature verification system characterizing dynamics of handwriting features[J]. EURASIP Journal on Advances in Signal Processing, 2013, 2013(1): 1-15.

[7] Igarza, Juan J., et al. Searching for an optimal reference system for on-line signature verification based on (x, y) alignment[J]. Biometric Authentication. Springer Berlin Heidelberg, 2004, 519-525.

[8] Al-Mayyan W, Own H S, Zedan H. Rough set approach to online signature identification[J]. Digital Signal Processing, 2011, 21(3): 477-485.

[9] Lopez-Garcia M, Ramos-Lara R, Miguel-Hurtado O, et al. Embedded System for Biometric Online Signature Verification[J]. Industrial Informatics, IEEE Transactions on, 2014, 10(1): 491-501.

[10] Cpałka K, Zalasiński M. On-line signature verification using vertical signature partitioning[J]. Expert Systems with Applications, 2014, 41(9): 4170-4180.

[11] Ortega-Garcia J, Fierrez-Aguilar J, Simon D. MCYT baseline corpus: a bimodal biometric database [J]. IEE Proceedings-Vision, Image and Signal Processing, 2003, 150(6): 395-401. 